\title{
Phase contrast in Simultaneous Topography and Recognition Imaging
}

\author{
M. Fuss ${ }^{a}$, E. Sahagún ${ }^{b}$, M. Köber ${ }^{a}$, F. Briones ${ }^{a}$, M. Luna ${ }^{a}$, and J. J. Sáenz ${ }^{b, c *}$ \\ ${ }^{a}$ Instituto de Microelectrónica de Madrid, Consejo Superior de Investigaciones Científicas, \\ Isaac Newton 8, 28760 Tres Cantos, Madrid, Spain \\ ${ }^{b}$ Departamento de Física de la Materia Condensada, Universidad Autónoma de Madrid, \\ 28049 Madrid, Spain
}

${ }^{c}$ Donostia International Physics Center (DIPC), Paseo Manuel Lardizabal 4, 20018 Donostia-San Sebastian, Spain

*Corresponding authors. Tel.: (+34) 9149738 04, fax: (+34) 9149739 61, e-mail: juanjo.saenz@uam.es (J.J. Sáenz); e-mail: mcfuss@imaff.cfmac.csic.es (M. Fuss)

\begin{abstract}
The operation of a force microscope in Simultaneous Topography and Recognition Imaging (TREC) mode is analyzed by means of numerical simulations. Both topography and recognition signals are analyzed by using a worm-like chain force as the specific interaction between the functionalized tip probe and the sample. The special feedback mechanism in this mode is shown to couple the phase signal to the presence of molecular recognition interactions even in absence of dissipation.
\end{abstract}

Keywords: Atomic Force Microscopy, Dynamic Force Microscopy, Molecular Recognition Imaging

\section{Introduction}

Since the beginnings of Atomic Force Microscopy (AFM), measurement techniques have steadily evolved leading to a growing field of successful applications in biology [1]. Force spectroscopy [2] (consisting in recording force vs. distance curves) represents an excellent method for studying molecular recognition, a key issue in many biological processes. However, until recently it was impossible to obtain independent recognition maps at the same resolution and imaging speed as the conventional maps of topography, lateral force, phase, etc. The introduction of TREC Imaging [3] provided a simple and fast Dynamic Force Microscopy mode capable of simultaneously recording images of topography and specific recognition between a functionalized tip probe and the sample. Among other applications, it has been used to observe protein movement within biomolecular complexes [4] and to localize receptors on cells [5]. 
As opposed to other dynamic force microscopy tools, phase contrast has not yet been explored in TREC imaging. Phase contrast images often provide significantly more contrast than the topographic image. Our main goal here is to discuss the possibility of phase contrast imaging in TREC mode. Although at fixed feedback amplitude, phase shift variations are often directly linked to energy dissipation processes [6-10], we will see that when using the special TREC feedback mechanism, phase shift couples to the presence of long-range molecular recognition interactions in absence of dissipation.

TREC is based on the spatial and temporal separation of localized attractive forces caused by recognition events and repulsive ones during contact. This is experimentally achieved by the separate evaluation of the oscillation minima and maxima when working with a heavily damped system (typically in liquid environment) in intermittent contact mode. Although there have been a few analytical approaches treating recognition imaging [11,12], very limited models as respects tip-sample interactions were used, and the special feedback loop, which is essential in TREC mode, was not taken into account.

[Figure 1: color on the web, black-and-white reproduction in print]

\section{Theory and Numerical Methods}

For simplicity, neither hydrodynamic damping of the cantilever or squeezing effects of the tip [13], nor higher eigenmode excitation [14,15] are taken into account here. We consider a point-mass model where the cantilever-tip system is treated as a damped, driven harmonic oscillator with additional terms describing tip-sample interactions. The equation of motion thus reads

$$
m \ddot{x}+\frac{m \omega_{R}}{Q} \dot{x}+k_{c} x=F_{0} \cos (\omega t)+F_{\text {int }}+F_{\text {rec }}
$$

where $\mathrm{x}$ is the tip's instantaneous position with respect to its equilibrium position, $t$ is time, $k_{c}$ is the cantilever's spring constant, $Q$ is the quality factor of the system, $F_{0}$ is the driving force, $\omega$ is the (angular) driving frequency, and $\omega_{R}=\omega_{0} / \sqrt{1+1 /\left(2 Q^{2}\right)}$ is the (angular) resonance frequency. The effective cantilever mass is calculated as $m=k_{c} / \omega_{0}{ }^{2}$, being $\omega_{0}$ the cantilever's eigenfrequency. Since recognition imaging is assumed to take place in a liquid environment and very close to the sample surface, low quality factors (0.5-6) are used. We distinguish two different types of tip-sample interactions. On the one hand, non-specific tipsample interactions are present everywhere on the sample and consist of attractive van-derWaals forces and Derjaguin-Muller-Toporov [16] contact repulsion:

$$
\begin{array}{ll}
F_{\text {int }}(d)=-\frac{H R}{6 d^{2}} & \left(d \geq a_{0}\right) \\
F_{\text {int }}(d)=-\frac{H R}{6 a_{0}^{2}}+\frac{4}{3} E^{*} \sqrt{R}\left(a_{0}-d\right)^{3 / 2} & \left(d<a_{0}\right)
\end{array}
$$

They are determined by the Hamaker constant $H$, the tip radius $R$, the instantaneous tipsample separation $d$, the effective elastic constant $E^{*}$ and an intermolecular distance $a_{0}$ which 
defines the onset of contact repulsion. On the other hand, specific recognition forces between the ligand tethered to the tip and its epitopes on the sample surface are continuously present while the tip is scanning over designated recognition sites. They are modelled using the worm-like chain model (WLC) which predicts [17]

$$
F_{\text {rec }}(d)=-\frac{k_{B} T}{L_{p}}\left[\frac{1}{4\left(1-d / L_{0}\right)^{2}}-\frac{1}{4}+\frac{d}{L_{0}}\right]
$$

( $k_{B}$ is Boltzmann's constant, $T$ is temperature) and which is known to adequately describe polymeric molecules such as poly(ethylen glycol) [18]. Note that this "recognition" force depends on the properties of the cross-linker used (contour length, $L_{0}$, and persistence length, $L_{\mathrm{p}}$ ) but not on the molecular recognition process itself which is here assumed to be stronger than the forces acting during an oscillation cycle. All simulations were carried out with peakto-peak oscillation amplitudes smaller than the tether length; accordingly no recognition bond ruptures have to be taken into account while the tip oscillates above one recognition site [19]. For integration of the resulting differential equation, a fifth-order Runge-Kutta algorithm employing Cash-Karp coefficients [20] was used. Results were obtained using the following parameters unless otherwise stated: $k_{c}=0.1 \mathrm{~N} / \mathrm{m}, Q=1, \omega=\omega_{0}=45 \mathrm{kHz}, H=1.5 \cdot 10^{-20} \mathrm{~J}$, $R=15 \mathrm{~nm}, E^{*}=50 \mathrm{GPa}$, surface energy $\gamma=0.0075 \mathrm{Jm}^{-2}, a_{0}=(H /(24 \pi \gamma))^{1 / 2}, T=293 \mathrm{~K}, L_{0}=$ $8 \mathrm{~nm}, L_{\mathrm{p}}=0.38 \mathrm{~nm}$ [18], free amplitude $=3 \mathrm{~nm}$, reduced (tapping) amplitude $=2.5 \mathrm{~nm}$ (outside binding sites).

\section{Results and Discussion}

Let us first examine the cantilever's response while scanning along a line containing both topographic features and recognition sites with the feedback loop turned off, that is, at a constant height (fig. 2a). It clearly shows the different influence of changes in topography or in the recognition behaviour. Whereas recognition sites (striped portions in fig. 2c) cause the oscillation maxima to decrease due to the attractive forces generated by the extended linker, changes in topography mostly affect the oscillation minima. This is in qualitative accordance with previous experimental results [3] and thus indicates that the model employed includes all the essential components for simulating recognition imaging. However, in order to efficiently distinguish contributions from topography and recognition events (and to keep the scanning tip at a constant distance from the sample), a feedback loop based on the deflection minima has to be employed [3]. Only then, the signal provided by the deflection maxima contains the true recognition information and the vertical piezo movement compensating the minima signal accurately represents topography, as can be seen in fig. $2 \mathrm{~b}$. The ability of the minima feedback to separate both signals was not observed to cease at least up to $Q=30$.

[Figure 2: color on the web, black-and-white reproduction in print]

Our next aim is to consider phase information instead of the deflection maxima for the detection of recognition sites. In order to be a good recognition detector, phase needs to be sensitive to recognition events but blind to topographic changes. Our model predicts that in absence of feedback, phase varies both as a consequence of corrugation and of recognition events. However, if the minima feedback is turned on, phase changes only due to the latter 
ones (fig. 3).

[Figure 3: color on the web, black-and-white reproduction in print]

Note that in our model, all interactions are conservative and changes in phase are not related to dissipation, as is traditionally interpreted. Instead, phase contrast is produced by the long range of the WLC interaction which creates a force gradient throughout the oscillation cycle. Thereby the resonance frequency of the cantilever is modified, much like it happens when long-range electrostatic forces are present. Additionally, the fact that minima feedback does not keep amplitude constant does further contribute to variations in phase which can be of the same or opposite sign as the ones caused by the first phenomenon. For both reasons, phase becomes sensitive to recognition.

In fig. 4a, the contrast in recognition signals, acquired from the oscillation maxima and from phase shift, are depicted for different quality factors. Within the range of Q studied, the maxima signal presents an increasing contrast for higher quality factors, while contrast in the phase signal peaks for low values (around 4.0 for the conditions shown).

[Figure 4: color on the web, black-and-white reproduction in print]

With the objective to enhance recognition detection, phase and maxima signal contrast has been studied as a function of the linear driving frequency $v$ (fig. $4 \mathrm{~b}$ ). For the frequencies studied, phase contrast ranges from $4^{\circ}$ to $-2^{\circ}$, whereas the maxima signal only changes between $1 \AA$ and $3.5 \AA$, suggesting that changes in phase will be much easier to measure. Contrast in the phase shift shows a maximum at the cantilever's eigenfrequency (which lies above resonance frequency in heavy damping conditions). For higher frequencies, contrast first diminishes and then adopts negative values. Note that although measurement conditions yielding zero contrast remove the possibility of recognition detection, sign inversion is not a problem for TREC imaging. In the maxima signal, contrast increases for higher driving frequencies.

Therefore, when recognition imaging is carried out using a low Q factor and driving the cantilever at its eigenfrequency, the AFM's phase signal should be considered for acquiring recognition information. If, however, only the maxima signal is to be used, it is preferable to drive the cantilever above its eigenfrequency in order to enhance signal contrast.

\section{Conclusions}

In summary, we have simulated TREC imaging by using a worm-like chain model for the recognition interaction, corresponding to the stretching of a cross-linker. This model reproduces experimental findings and predicts that the feedback mechanism causes phase information to be coupled to the presence of molecular recognition interactions. Phase information can provide better recognition sensitivity than the maxima signal and could be used either independently or in correlation with the latter in order to detect binding sites. The recording of recognition maps based on phase contrast is especially useful when working with 
low quality factors at the natural frequency. However, if only the maxima signal is to be used, recognition sensitivity can be enhanced by driving the cantilever above its eigenfrequency. We believe that exploring the phase contrast in recognition imaging experimentally will be of great interest and hope that the predictions made by our model will be tested in the laboratory soon.

Finally, we would like to emphasize that the usage of a simple model was aimed at understanding the essential functionality of TREC mode imaging by including its distinctive features alongside only the most important general elements. This basic approach helps clarity and facilitates studying recognition effects in dynamic AFM separated from other phenomena that may be commonly encountered. However, it is not expected to yield exact absolute phase values since some effects or special conditions not considered herein (e.g., hydrodynamic damping included in continuum models [13] or excitation of higher flexural modes as recently described [15]) might alter them. Quantitative comparison with experiments under physiological conditions would also require the use of the Derjaguin-Landau-VerweyOverbeek (DLVO) theory [21-23,14] to account for the electrostatic interactions in solution. However, including a more realistic description of the interaction does not alter the essential conclusions on phase contrast in TREC imaging.

\section{Acknowledgements}

M.F. acknowledges the hospitality offered to her by P. Hinterdorfer's group at the Institute of Biophysics, University of Linz, during the completion of this work.

This work was supported by the Spanish MICINN through the projects CTQ2005-07993C0202/BQU, NAN2004-09125-C07-02, FIS2006-11170-C02-02, and FIS2005-05137, the Comunidad de Madrid Project Microseres-MC and by the EU IP "Molecular Imaging" (LSHG-CT-2003-503259). M.F. acknowledges financial support from the MICINN. M.K. acknowledges an I3P scholarship. M.L. acknowledges financial support from the MICINN through the Ramón y Cajal program.

\section{References}

[1] N. Jalili, K. Laxminarayana, Mechatronics 14 (2004) 907-945.

[2] J. Zlatanova, S. M. Lindsay, S. H. Leuba, Progress in Biophysics and Molecular Biology 74 (2000) 37-61.

[3] C. Stroh, A. Ebner, M. Geretschläger, G. Freudenthaler, F. Kienberger, A.S.M. Kamruzzahan, S.J. Smith-Gill, H.J. Gruber, P. Hinterdorfer, Biophys. J. 87 (2004) 1981-1990.

[4] R. Bash, H. Wang, C. Anderson, J. Yodh, G. Hager, S.M. Lindsay, D. Lohr, FEBS Letters 580 (2006) 4757-4761.

[5] L.A. Chtcheglova, J. Waschke, L. Wildling, D. Drenckhahn, P. Hinterdorfer, Biophys. J. 93 (2007) L11-L13.

[6] J.P. Cleveland, B. Anczykowski, A.E. Schmid, V.B. Elings, Appl. Phys. Lett. 72 (1998) 2613-2615. 
[7] J. Tamayo, R. García, Appl. Phys. Lett. 73 (1998) 2926-2928.

[8] P.D. Ashby, C.M. Lieber, J. Am. Chem. Soc. 127 (2005) 6814-6818.

[9] E. Sahagún, P. García-Mochales, G.M. Sacha, J.J. Sáenz, Phys. Rev. Lett. 98 (2007) 176106.

[10] M. Köber, E. Sahagún, M. Fuss, F. Briones, M. Luna, J.J. Sáenz, phys. stat. sol. (RRL) 2 (2008) 138-140.

[11] H. Schindler, D. Badt, P. Hinterdorfer, F. Kienberger, A. Raab, S. Wielert-Badt, V.Ph. Pastushenko, Ultramicroscopy 82 (2000) 227-235.

[12] V.Ph. Pastushenko, P. Hinterdorfer, F. Kienberger, C. Borken, Hansgeorg Schindler, Single Mol. 1 (2000) 165-170.

[13] Y. Song, B. Bhushan, Ultramicroscopy 107 (2007) 1095-1104.

[14] S. Basak, A. Raman, Appl. Phys. Lett. 91 (2007) 064107.

[15] X. Xu, J. Melcher, S. Basak, R. Reifenberger, A. Raman, Phys. Rev. Lett. 102 (2009) 060801.

[16] B.V. Derjaguin, V.M. Muller, Y.P. Toporov, J. Colloid Interface Sci. 53 (1975) 314326.

[17] C. Bustamante, J.F. Marko, E.D. Siggia, S. Smith, Science 265 (1994) 1599-1600.

[18] F. Kienberger, V. Pastushenko, G. Kada, H. Gruber, C. Riener, H. Schindler, P. Hinterdorfer, Single Mol. 1 (2000) 123-128.

[19] A. Ebner, F. Kienberger, G. Kada, C. Stroh, M. Geretschläger, A.S.M. Kamruzzahan, L. Wildling, W.T. Johnson, B. Ashcroft, J. Nelson, S.M. Lindsay, H.J. Gruber, P. Hinterdorfer, ChemPhysChem 6 (2005) 897-900.

[20] J.R. Cash, A.H. Karp, ACM Transactions on mathematical software 16 (1990) 201-222.

[21] B. Derjaguin, Trans. Faraday Soc. 36 (1940) 203.

[22] B.V. Derjaguin, L.D. Landau, Acta Physicochimica (URSS) 14 (1941) 633.

[23] E.J.W. Verwey, J.Th.G. Overbeek, Theory of the Stability of Lyophobic Colloids, Elsevier

Publishing

Co.,

Amsterdam,

1948. 


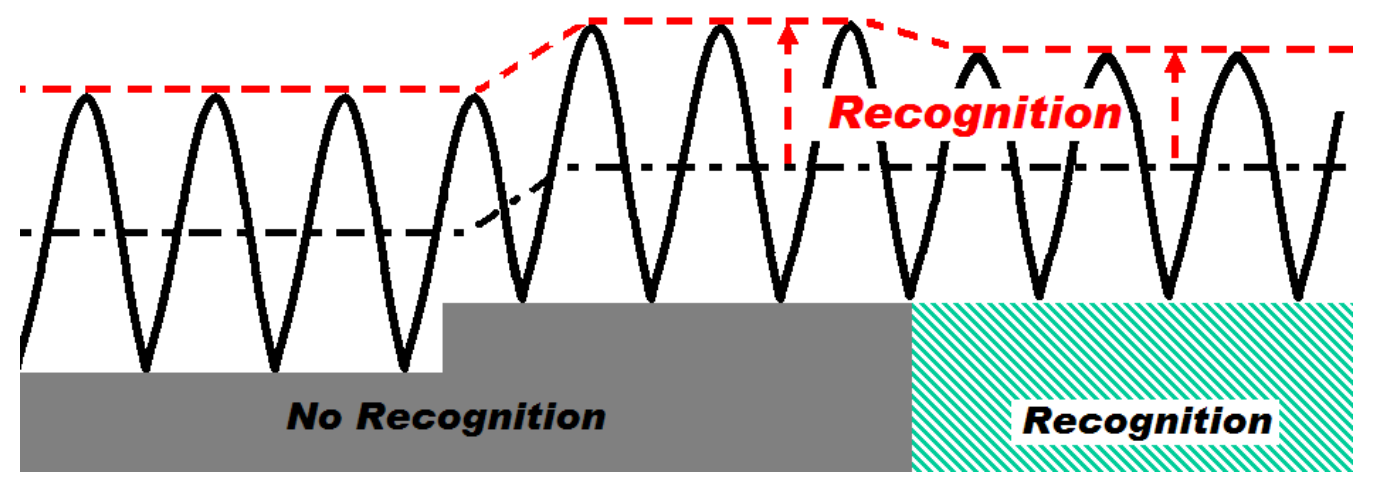

Figure 1. Sketch illustrating the collection of separate topography and recognition signals in TREC mode. The striped portion of the substrate indicates the presence of specific molecular interaction.

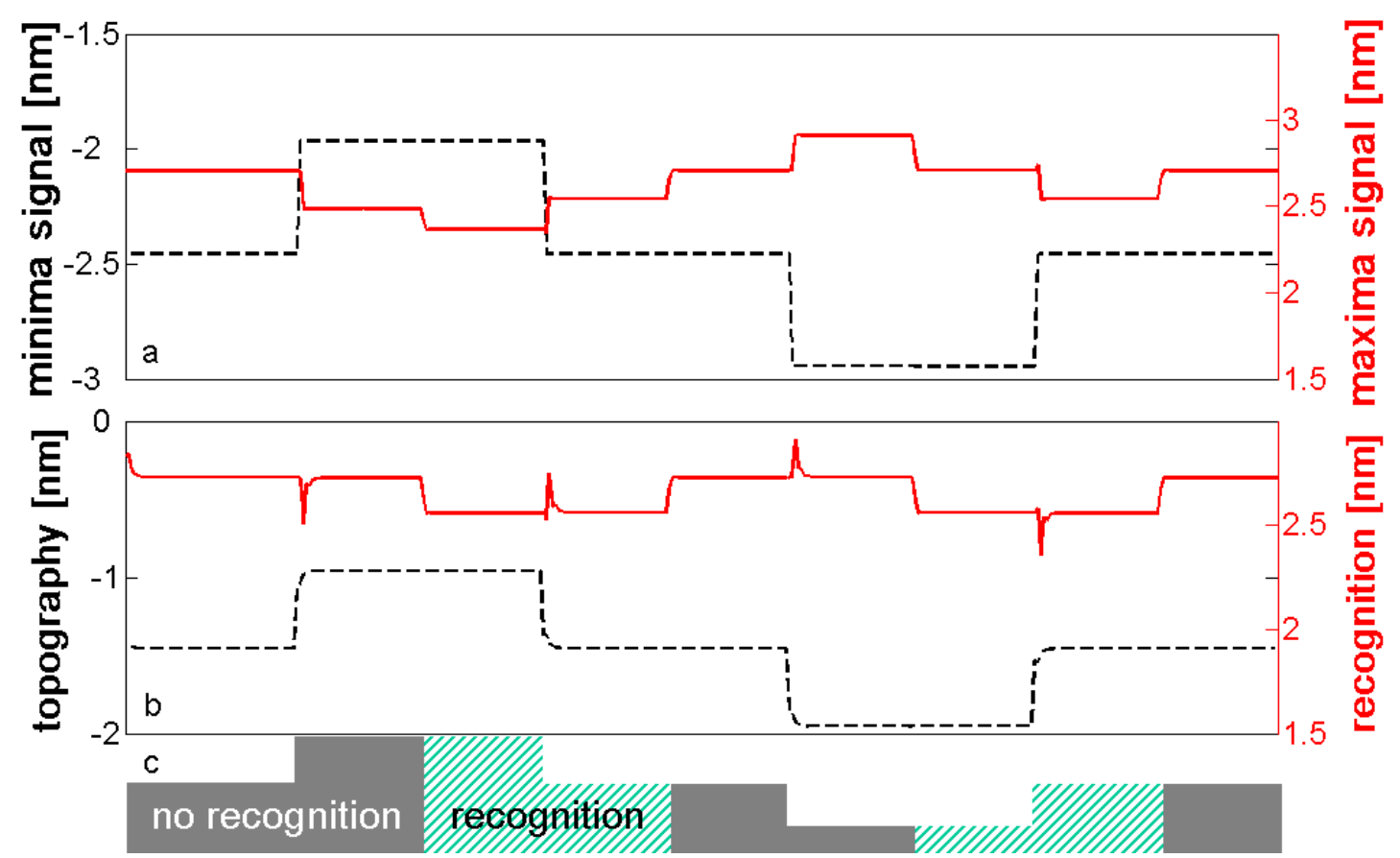

Figure 2. Simulated scan line of an area containing both topographic and recognition features as sketched in (c), without (a) and with (b) feedback. In (b), it can be seen that the topography signal (dashed line, left scale) represents an accurate measure of the sample's height (all steps are $0.5 \mathrm{~nm}$ high), whereas the recognition signal (solid line, right scale) identifies the epitopes on the sample. 


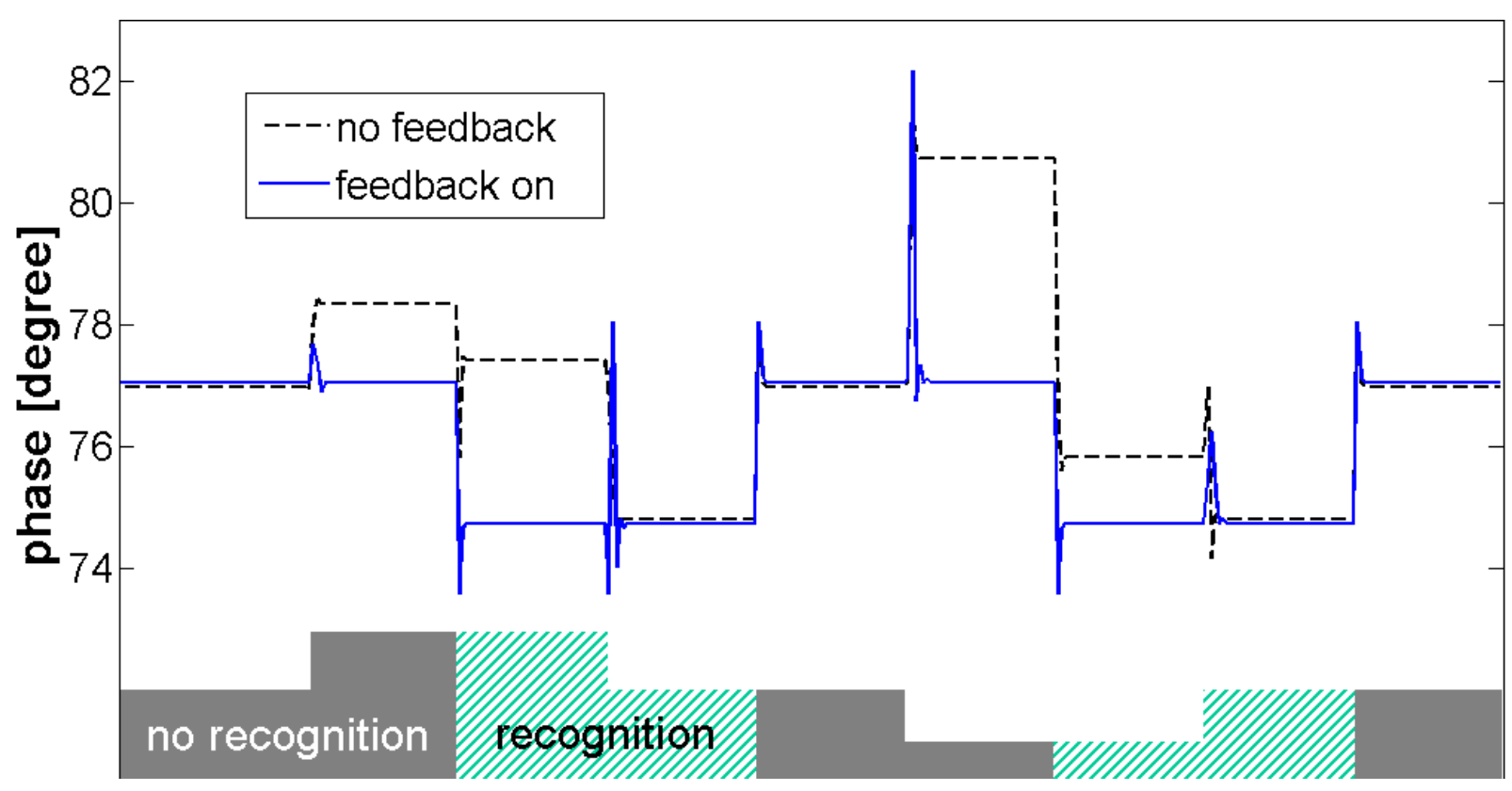

Figure 3. Phase shift between driving force and tip deflection with (solid line) and without (dashed line) feedback for a scan line of an area containing both topographic and recognition features (same case as shown in fig. 2). When feedback is performed to keep oscillation minima at a fixed distance from the tip's rest position, phase contrast is coupled to the presence of binding sites (striped portions in sketch). 

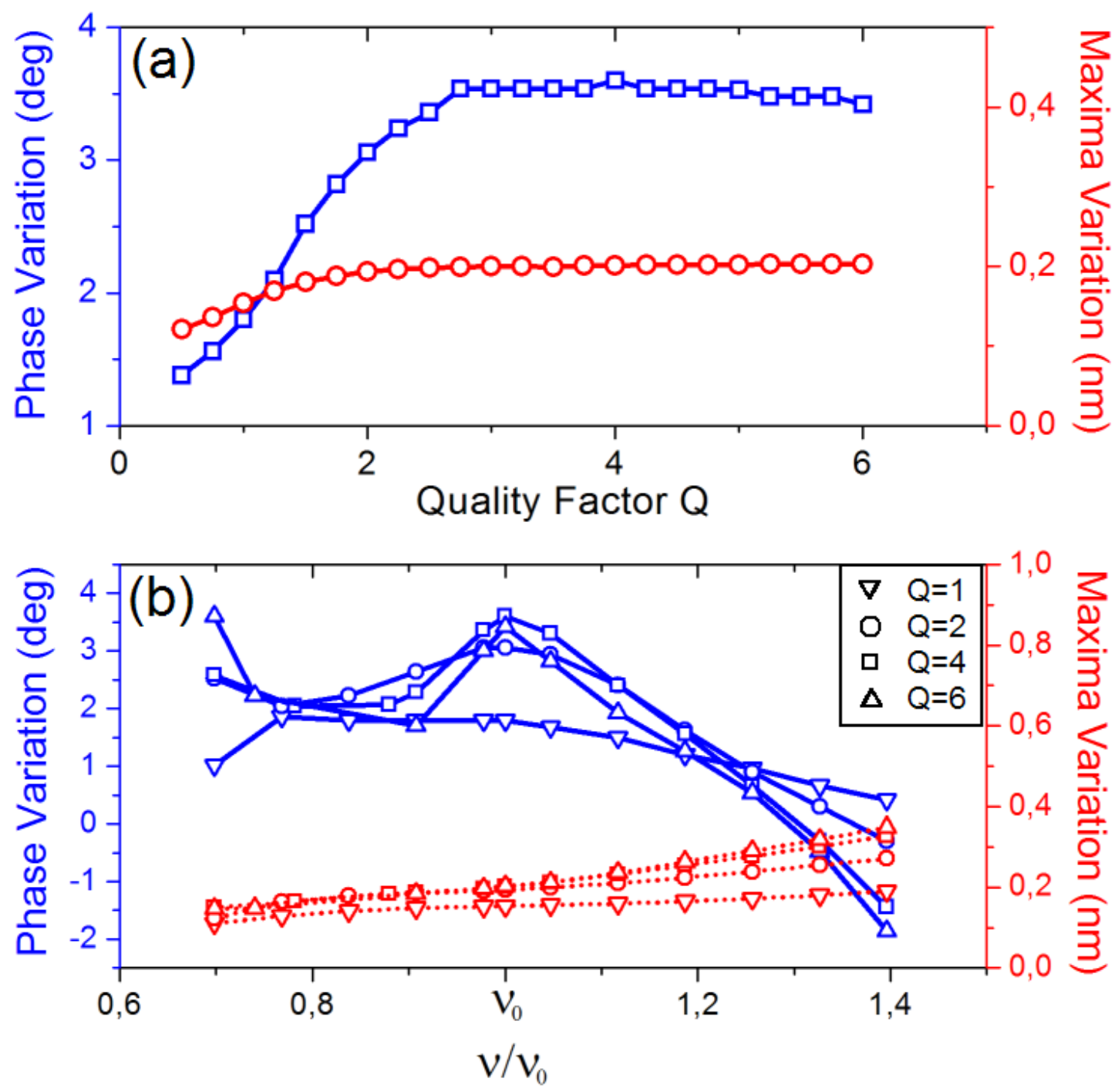

Figure 4. Contrast (signal change with vs. without recognition interaction) obtained in oscillation maxima (dashed line, right scale) and phase (solid line, left scale) signals as a function of quality factor (a) and driving frequency (b). 